\title{
Simulating Surface Charge Dynamics
}

\author{
G. Callender, K. F. Goddard and P. L. Lewin \\ The Tony Davies High Voltage Laboratory \\ School of Electronics and Computer Science \\ University of Southampton \\ Southampton, SO17 1BJ, UK
}

\begin{abstract}
Surface charge dynamics can play an important role in electric field distributions in both AC and DC insulation systems. In this paper the theory behind surface charge dynamics is introduced with a particular focus on the calculation of surface divergence; an operator which is used to determine movement of charge constrained to a surface. A detailed discussion of the model implementation is provided and validated against an analytical solution. Surface charge dynamics in AC and DC insulation systems are then investigated. Assuming the measurement data in the literature is representative it is demonstrated that surface currents have the capability to significantly alter electric field distributions in voids, even over the timescales of an AC cycle.
\end{abstract}

Index Terms - surface charge density, charge transport, finite element methods

\section{INTRODUCTION}

INCREASING operating voltages are leading to insulation materials being subjected to rising electric fields. In AC systems this can increase the likelihood of partial discharge (PD) activity in insulation defects. PD activity deposits surface charge on insulating materials, such that it quenches each discharge [1]. However, once the voltage polarity is reversed in the next half cycle it will enhance the electric field promoting further discharges which will deposit charge of opposite polarity. Understanding the movement and decay of surface charge between discharges, which may be significant in defects with a highly conductive surface, is important for understanding discharge activity [2]. For DC systems, which are being increasingly used in long distance transmission networks, the applied voltage is typically held at a fixed magnitude and polarity and as surface charge due to PD cannot be neutralized by PDs of opposite polarity; it can be removed only by onward bulk conduction. In practice switching events and harmonics will also play an important role [3]. Furthermore, as the polarity of the applied voltage is held constant in DC systems, charge injection may lead to significant accumulation of space charge within insulation domains and surface charge at insulation interfaces. Space charge within DC systems can enhance electric fields in certain parts of insulation systems which can lead to the premature failure of electrical equipment [3].

Within the electrical engineering community the majority of the research on surface charge dynamics can be traced back to work performed by McAllister in the 1980's and 1990's [4, 5, 6]. This research predominantly focused on analytical calculations for relatively simple systems. More recently measurement and simulation work has been performed to investigate surface charge dynamics on flat insulator surfaces [7, 8, 9]. It has been demonstrated that sustained PD activity can lead to an increase in surface conductivity due to the formation of conductive byproducts due to discharge activity [10]. More recent work has mapped surface conductivity as a function of position at void surfaces, demonstrating that discharges may cause inhomogeneous damage to void surfaces [11]. Moving forwards it will be necessary to consider more complex insulator geometries that may be present in operational high voltage plant. The dependency of surface conductivity on tangential electric field strength and temperature, which is likely to be the norm rather than the exception [4], must also be considered. Simulations can be used to inform such experimental investigations.

The purpose of this paper is to present a method to calculate surface charge density dynamics. Much of the original literature on the subject, [6], does not lend itself to simulation techniques using standard simulation tools. This work has adopted equivalent analytical methodology, [12], which allows the use of a commercial package to provide a numerical solution which facilitates simulating surface charge dynamics for a range of systems. The approach presented here does not distinguish between different types of charge carriers, it is instead focusing on solving for the charge densities. An alternative approach would be to use a drift diffusion model of space charge, e.g. [13], adapted for the movement of charge constrained to a surface. Such a model requires a large number of parameters, such as charge carrier mobilities and recombination coefficients, which are difficult to determine experimentally. In this work this complexity is ignored and the only dependent variable at an interface is the surface charge density. All surface charge transport is assumed to be captured in a surface conductivity with a corresponding surface current density. The discussion in this paper on the implementation required to calculate the surface divergence of surface current densities would be of relevance to any future work which uses drift diffusion models of different charge carriers constrained to a surface. 


\section{THEORY}

In this section the theory behind surface charge density dynamics is introduced. All of the fundamental theory is based on electrostatics and the conservation of electronic charge. With

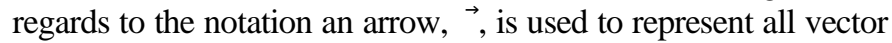
operators and quantities for the sake of clarity.

\subsection{GOVERNING EQUATIONS}

For insulation systems under consideration it is generally appropriate to use the electrostatic equation to determine electric field $\vec{E}$ as follows

$$
\vec{\nabla} \cdot \vec{E}=\frac{\rho}{\varepsilon_{0} \varepsilon_{\mathrm{r}}}
$$

where $\rho$ is the volume charge density, $\varepsilon_{0}$ is the permittivity of free space and $\varepsilon_{r}$ is the relative permittivity of the insulation. The conservation of charge in the bulk requires that

$$
\frac{\partial \rho}{\partial t}+\vec{\nabla} \cdot \vec{J}=0
$$

where $\vec{J}$ is the current density. At interfaces between different dielectric materials the conservation of charge requires

$$
\begin{aligned}
& \sigma=\vec{n} \cdot\left(\varepsilon_{0} \varepsilon_{\mathrm{r}+} \vec{E}_{+}-\varepsilon_{0} \varepsilon_{\mathrm{r}-} \vec{E}_{-}\right) \\
& \frac{\partial \sigma}{\partial t}+\vec{\nabla}_{S} \cdot \vec{K}+\vec{n} \cdot\left(\vec{J}_{+}-\vec{J}_{-}\right)=0
\end{aligned}
$$

where $\sigma$ is the surface charge density, $\vec{\nabla}_{S}$ is the surface divergence, $\vec{K}$ is the surface current density and $\vec{n}$ is a normal vector on the surface. The \pm subscripts denote evaluation on different sides of the surface with $\vec{n}$ pointing into the positive side.

\subsection{SURFACE DIVERGENCE}

In order to simulate surface charge density dynamics, Equation (4), it is necessary to calculate the surface divergence of the surface current density $\vec{K}$. The definition of surface divergence of a vector field $\vec{F}$ that one typically finds in the electrical engineering literature is

$$
\vec{\nabla}_{S} \cdot \vec{F}=\lim _{\Delta A \rightarrow 0}\left(\frac{1}{\Delta A} \oint_{C} \vec{F} \cdot \vec{m}\right)
$$

where $C$ is a closed curve on the surface with an outward facing normal vector $\vec{m}$ enclosing an area $\Delta A$. It is important to understand that this is not equivalent to the typical three dimensional divergence.

Equation (5) as written is difficult to implement numerically as it requires line integrals to be evaluated over mesh elements. Arnoldus derived a preferable formulation in [12] where the surface divergence is written as

$$
\vec{\nabla}_{S} \cdot \vec{F}=\frac{1}{\sqrt{\operatorname{det} G}}\left[\frac{\partial}{\partial u}\left(F^{u} \sqrt{\operatorname{det} G}\right)+\frac{\partial}{\partial v}\left(F^{v} \sqrt{\operatorname{det} G}\right)\right]
$$

where det is the determinant; $G$ is the surface metric; $u$ and $v$ are the surface parameters; and $F^{u}$ and $F^{v}$ are the contravariant components of $\vec{F}$. Equation (6) is a differential equation which is more amenable to standard numerical techniques. A derivation of Equation (6) from Equation (5) is provided in [12]. Earlier work by McAllister, [4], derived similar expressions. However, they do not appear to have permeated the literature and Equation (5) is typically used as written $[2,7]$.

To calculate the surface metric $G$ the surface $S$ needs to be defined first as

$$
\vec{r}=x(u, v) \vec{e}_{x}+y(u, v) \vec{e}_{y}+z(u, v) \vec{e}_{z}
$$

where $\vec{r}$ is the coordinate vector of points on the surface; $x, y$ and $z$ are functions of the surface parameters; and $\vec{e}_{x}, \vec{e}_{y}$ and $\vec{e}_{z}$ are the standard Cartesian unit vectors. The surface is spanned by two tangent vectors $\vec{c}_{u}$ and $\vec{c}_{v}$

$$
\begin{aligned}
& \vec{c}_{u}=\frac{\partial \vec{r}}{\partial u} \\
& \vec{c}_{v}=\frac{\partial \vec{r}}{\partial v} .
\end{aligned}
$$

A schematic showing variables of interest for an arbitrary surface is provided in Figure 1.

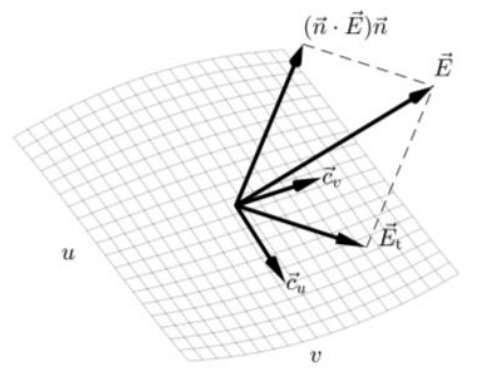

Figure 1. Schematic showing surface variables, electric field and tangential electric field for an arbitrary surface parameterized by $u$ and $v$.

The surface metric $G$ is defined as

$$
G=\left(\begin{array}{ll}
\vec{c}_{u} \cdot \vec{c}_{u} & \vec{c}_{u} \cdot \vec{c}_{v} \\
\vec{c}_{u} \cdot \vec{c}_{v} & \vec{c}_{v} \cdot \vec{c}_{v}
\end{array}\right)
$$

the contravariant tangent vectors are defined as

$$
\begin{aligned}
& \vec{c}^{u}=G_{u u}^{-1} \vec{c}_{u}+G_{u v}^{-1} \vec{c}_{v} \\
& \vec{c}^{v}=G_{u v}^{-1} \vec{c}_{u}+G_{v v}^{-1} \vec{c}_{v}
\end{aligned}
$$

where $G^{-1}$ is the inverse of $G$. The contravariant tangent vectors are used to calculate the contravariant components of $\vec{F}$

$$
F^{u}=\vec{c}^{u} \cdot \vec{F} \text { and } F^{v}=\vec{c}^{v} \cdot \vec{F} \text {. }
$$

The calculations required to solve Equation (6) may appear excessive. However, the majority are dependent on the surface geometry and as such are unlikely to change during a given simulation. Furthermore, it allows the surface divergence to be evaluated numerically with greater ease as it is a differential equation.

A final point regarding surface divergence is that for the special case of flat surfaces, i.e. surfaces which can defined as

$$
\vec{r}=u \vec{e}_{x}+v \vec{e}_{y}
$$

for a given coordinate system, the surface divergence reduces to the standard two dimensional divergence

$$
\vec{\nabla}_{S} \cdot \vec{F}=\frac{\partial F_{x}}{\partial x}+\frac{\partial F_{y}}{\partial y} \text {. }
$$

where $F_{x}$ and $F_{y}$ are the $x$ and $y$ components of $\vec{F}$ respectively. This result is also mentioned in [12]. 


\subsection{ELECTRICAL CONDUCTIVITY}

Assuming that the material is Ohmic the current density $\vec{J}$ and the surface current density $\vec{K}$ can be written as

$$
\begin{aligned}
& \vec{J}=\gamma \vec{E} \\
& \vec{K}=\Gamma \vec{E}_{\mathrm{t}}
\end{aligned}
$$

where $\gamma$ is the volume conductivity, $\Gamma$ is the surface conductivity and $\vec{E}_{\mathrm{t}}$ is the tangential electric field which can be calculated as

$$
\vec{E}_{\mathrm{t}}=\vec{E}-(\vec{n} \cdot \vec{E}) \vec{n}
$$

where $\vec{n}$ is the normal unit vector to the surface. It is worth noting that the tangential component of the electric field is independent of the side of the surface used to evaluate it. This is in contrast to the electric field normal to the surface, which may be discontinuous due to the changing relative permittivity and the presence of surface charge.

By defining an electrical conductivity for a given material, be it in the bulk or at an interface, it is implicitly assumed that all of the mechanisms of conduction can be treated using a single lumped parameter, the conductivity, which is not necessarily accurate [14]. However, more complex models of conduction, such as driftdiffusion space charge models, typically introduce additional parameters which can be difficult to quantify. Electrical conductivity is therefore treated as a meaningful physical property in this paper. In the examples considered here it is mostly assumed to be independent of temperature and electric field strength for the sake of simplicity; no changes to the implementation or the theory introduced in Sections 2.1 and 2.2 would be required to introduce such dependencies. To remain physically sensible it would be required that the surface conductivity is dependent on the magnitude of the tangential electric field, rather than the full electric field magnitude which will be discontinuous across a boundary of materials with different electrical properties. Furthermore, as the charge carriers are confined to the surface, there would be no reason for the normal field component to have any influence.

It is interesting to note that if the bulk conductivity $\gamma$ is treated as homogeneous then the conservation of charge, Equation (2), prohibits the production of space charge $\rho$. This can be seen by substituting Equation (16) into Equation (2), which results in

$$
\frac{\partial \rho}{\partial t}+\gamma \vec{\nabla} \cdot \vec{E}+\vec{E} \cdot \vec{\nabla} \gamma=0
$$

Substituting Equation (1) into Equation (19), and using the fact that homogeneous conductivity means $\vec{\nabla} \gamma=\overrightarrow{0}$, it follows that

$$
\frac{\partial \rho}{\partial t}+\frac{\gamma \rho}{\varepsilon_{0} \varepsilon_{\mathrm{r}}}=0
$$

Assuming $\rho=0$ at some time before the application of an applied voltage then $\rho$ must remain zero always in order to satisfy Equation (20).

\section{IMPLEMENTATION}

The simulations reported in this paper were all implemented in Comsol Multiphysics version 5.5. The default numerical solvers were found to be adequate for all of the considered scenarios. All of the models constructed for the paper were two-dimensional, both slice and axisymmetric simulations have been considered. The reason for this is two-fold. Firstly, the computational cost of three-dimensional models is prodigious as relatively fine meshes are required at boundaries in order to resolve surface charge dynamics. In many insulation systems symmetries can be exploited to produce representative twodimensional simulations which can still be used to provide insight into the operational three-dimensional system. Secondly, the implementation framework that is introduced in this paper could only be robustly applied to two-dimensional systems. Alternatives required coupling Comsol Multiphysics with Matlab and bespoke calculation, which is not in accordance with aims of the paper which is presenting an approach which is accessible for a more general user.

The governing equations which must be solved are Equations (1), (2), (3) and (4). Two coupled model components were used in each Comsol model. A two dimensional component was used to solve Equations (1) and (2) for electric potential $V$ and space charge density $\rho$ respectively in physical space. (1) is solved using the default electrostatics library, (2) was solved using a Domain Ordinary Differential Equations (ODEs) interface. External boundary conditions for $V$ are either set to fixed equipotential values, or to zero electric field normal to the boundary. At internal boundaries the continuity of electric potential and tangential electric field strength is enforced, the influence of surface charge is considered as per Equation (3).

The one dimensional component was used to solve Equation (4) using a one dimensional Domain ODEs interface. In order to calculate

$$
\frac{\partial}{\partial u}\left(F^{u} \sqrt{\operatorname{det} G}\right)
$$

it was found be necessary to solve for a dependent variable $\zeta$ where

$$
\frac{\partial \zeta}{\partial t}=F^{u} \sqrt{\operatorname{det} G}
$$

and then solve for $\sigma$ separately where

$$
\sqrt{\operatorname{det} G} \frac{\partial \sigma}{\partial t}+\frac{\partial^{2} \zeta}{\partial t \partial u}+\sqrt{\operatorname{det} G} \vec{n} \cdot\left(\vec{J}_{+}-\vec{J}_{-}\right)=0 .
$$

Transition maps between the one and two dimensional model components were implemented using Comsol's general extrusion operators. The map from the one dimensional model component to the two dimensional model component is inherent from the parameterization of the surface, Equation (7).

In terms of numerical settings the shape order of the electric potential $V$ at boundaries where surface charge dynamics are to be calculated must be at least cubic. The dependent variable $\zeta$, which depends on the tangential electric field can then be solved using quadratic order elements and the surface charge density $\sigma$ can be solved for using linear order elements. If the shape order was not incremented in this manner between the governing equations spurious numerical oscillations were observed. The maximum mesh element size was controlled at boundaries where surface charge dynamics were calculated and within the two dimensional domains. Mesh refinement was performed such that the difference in the dependent variables at locations of interest was less than $5 \%$ when both the maximum element size and time step size were halved. 


\section{COMPARISON WITH ANALYTICAL CALCULATIONS}

In this section a simple system is considered which has a known analytical solution both as a check of the model implementation and to provide a simple example for the reader and to provide confidence in using the model to investigate more complex systems. The system consists of a three dimensional infinite space containing a spherical void of radius $R$ and permittivity 1 . The void is surrounded by a material with a different permittivity, $\varepsilon_{\text {ra }}$. Initially two point charges of equal magnitude $q$ and opposite polarity are placed at antipodal points on the sphere. The initial state of the system is shown in Figure 2.

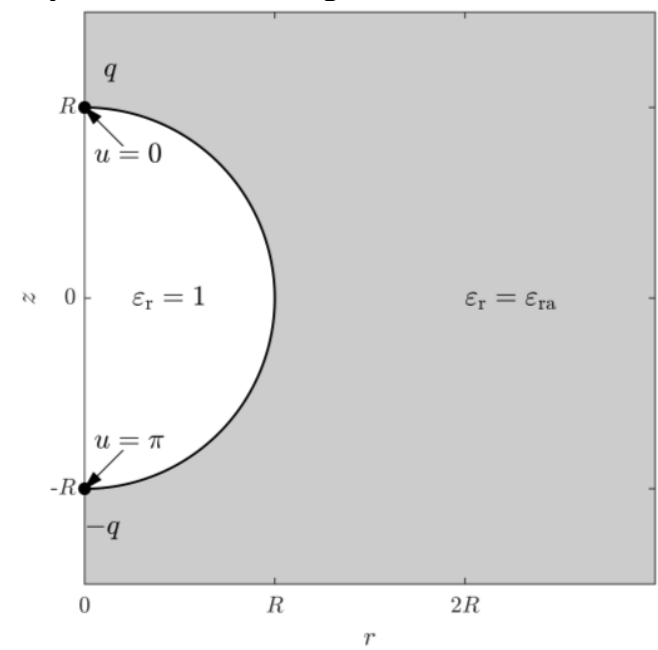

Figure 2. Schematic of the initial state of a system with point charges, with magnitude $q$, at antipodal points on a spherical void with a relative permittivity of 1 , enclosed within an infinite volume of homogenous dielectric material with a relative permittivity of $\varepsilon_{\text {ra }}$. The system is axisymmetric with respect to an axis between the charges. The axisymmetric surface is parameterized by the polar angle $u$.

The sphere is parameterized as follows

$$
\vec{r}=R \cos v \sin u \vec{e}_{x}+R \sin v \sin u \vec{e}_{y}+R \cos u \vec{e}_{z}
$$

where $v \in[0,2 \pi), u \in[0, \pi]$, and with the point charges located on the $z$ axis. Assuming that the bulk conductivity is negligible $(\gamma=0)$ the surface charge density can be calculated analytically to be

$$
\sigma=2 \sigma_{0} \sum_{n=0}^{\infty}(4 n+3) P_{2 n+1}(\cos u) \exp \left(g(n) \frac{t}{\tau}\right)
$$

where $\sigma_{0}=q / 4 \pi R^{2}, \tau=\varepsilon_{0} R / \Gamma, P_{n}$ is a Legendre polynonmial of degree $n$ and

$$
g(n)=\frac{-(2 n+1)(2 n+2)}{(2 n+2) \varepsilon_{\mathrm{ra}}+(2 n+1)} .
$$

A full derivation of Equation (25) can be found in [5]. $\tau$ is a variable introduced in the analytical calculations but it may also be viewed as a characteristic time scale of the surface charge dynamics.

A numerical simulation of the same system is then constructed. This requires solving Equation (4). Using the parameterization of the surface in Equation (24) the following variables can be calculated

$$
\operatorname{det} G=R^{4} \sin ^{2} u
$$

$$
\begin{aligned}
\vec{c}^{u} & =\frac{\cos v \cos u}{R} \vec{e}_{x}+\frac{\sin v \cos u}{R} \vec{e}_{y}-\frac{\sin u}{R} \vec{e}_{z} \\
\vec{c}^{v} & =-\frac{\sin v}{R \sin u} \vec{e}_{x}+\frac{\cos v}{R \sin u} \vec{e}_{y} .
\end{aligned}
$$

The model is axisymmetric, and as such will calculate the tangential electric field in terms of $r$ and $z$ coordinates. The $x$ and $y$ components of the tangential electric field are

$$
\begin{aligned}
& E_{\mathrm{t} x}=E_{\mathrm{t} r} \cos v \\
& E_{\mathrm{t} y}=E_{\mathrm{t} r} \sin v .
\end{aligned}
$$

Due to symmetry the value of $v$ used in the simulations will not influence the results and it is therefore set to zero. The contravariant components of the surface current density, $K^{u}$ and $K^{v}$, can then be calculated from the tangential electric field. The transition used to map from the two dimensional model component to the one dimensional model component is

$$
u=\frac{\pi}{2}-\tan ^{-1} \frac{z}{r} \text {. }
$$

The physical parameters $R, \sigma_{0}$ and $\tau$ are set to 1 for simplicity. The relative permittivity of the surrounding material is set to 4 , and is treated as an infinite element domain in order to match the analytical arrangement. At the far field boundary of the infinite element domain the electric potential is set to zero. Symmetry boundary conditions are employed on the $r=0$ axis.

The initial condition of the analytical calculation of two point charges is equivalent to a surface charge density distribution consisting of Dirac delta functions. This is not appropriate as an initial condition for the simulation as it requires an infinitely large surface charge density at the point charge locations and a surface charge density of zero everywhere else. Instead the simulation was initialized using the analytical solution at $t=0.5 \tau$, at which time, being after $t=0$, the surface charge density is a smooth finite function on the surface of the sphere. When evaluating the analytical solution, Equation (25), it is necessary to truncate the summation after a suitable number of terms. For the calculations performed here 100 terms were used. A comparison between the analytical solution and the numerical simulation is provided in Figure 3 . The percentage difference between the analytical and numerical solution is below $1 \%$ for all surface charge density magnitudes above $\sigma_{0}$.

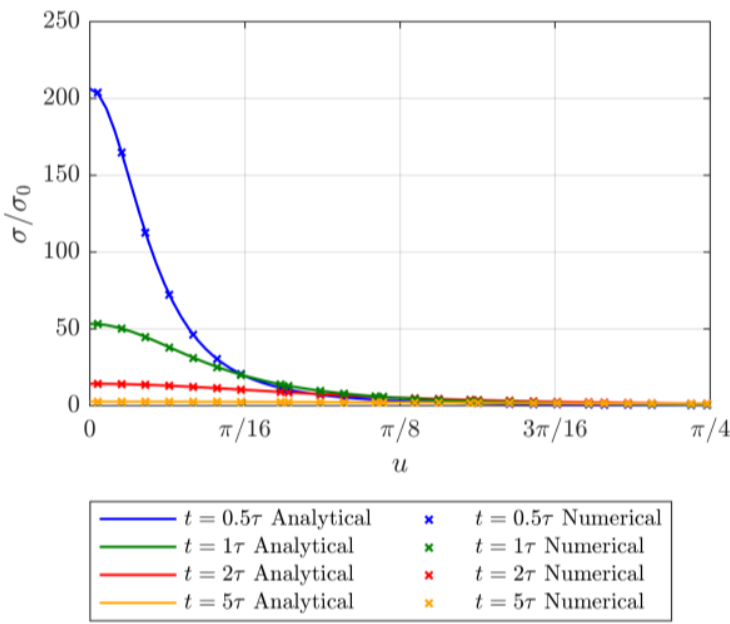

Figure 3. Analytical solution and numerical calculation at different time instances for $u \in[0, \pi / 4]$. 
The agreement between the analytical solution and the simulation is excellent, which validates the implementation of the model. The reader is strongly recommended to make use of these analytical calculations when developing models of surface conduction. The remainder of the paper demonstrates the potential influence of surface conduction for AC PD systems and DC insulation systems.

\section{DECAY OF DEPOSITED CHARGE IN AC PD SYSTEMS}

PDs occurring within gaseous voids will deploy charge at the void boundary which will oppose the electric field that initiated the discharge and quench it [15]. Between discharges the bipolar charge distributions will undergo neutralization due to surface conduction. Indeed the motivation of the analytical calculations in [5], which were used in the previous section, was to provide insight into this process. In this section systems which are more physically realistic than the analytical system are considered. Both are axisymmetric, the model geometries are provided in Figures 4 and 5 .

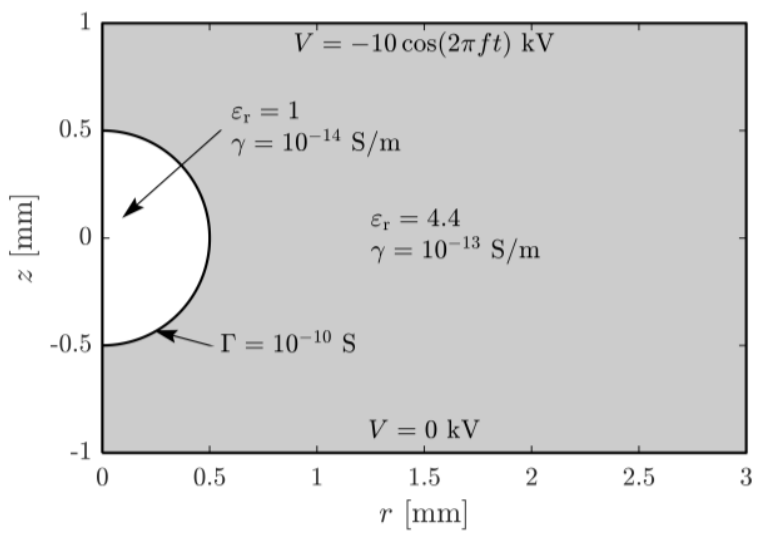

Figure 4. Axisymmetric model geometry for a spherical air void in epoxy resin. Electrical properties and the axial electric potential boundary conditions are also provided.

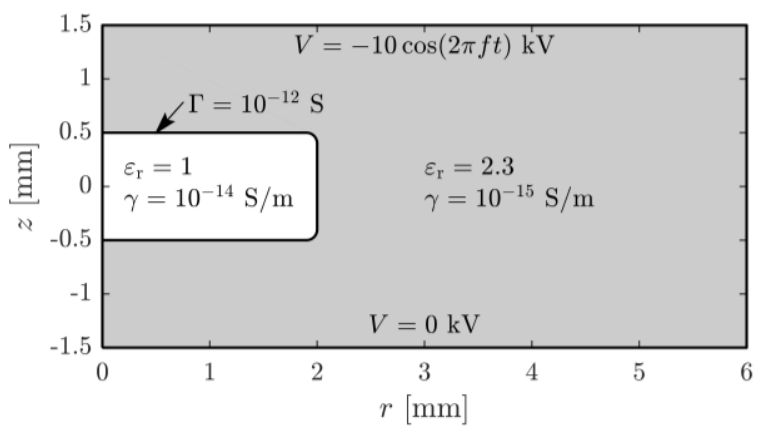

Figure 5. Axisymmetric model geometry for a cylindrical air void in LDPE. A fillet, with a radius of $0.1 \mathrm{~mm}$, has been applied at the corners of the cylindrical void to prevent field singularities. Electrical properties and the axial electric potential boundary conditions are also provided.

The systems are intended to be representative of typical PD experimental arrangements. Spherical voids in epoxy $\left(\varepsilon_{r}=\right.$ 4.4) can be fabricated by injecting air into the resin before curing, and have been investigated in the literature [2, 15]. Cylindrical voids in $\operatorname{LDPE}\left(\varepsilon_{r}=2.3\right)$ are typically fabricated using three sheets pressed together with a hole drilled in the central sheet [16]. Selecting appropriate values for conductivities of the dielectric materials, the air, and the surface conductivity is non-trivial, and would in practice need to be determined experimentally for each setup. For the illustrative purposes of this paper the volume conductivity is set to $10^{-13} \mathrm{~S} / \mathrm{m}$ [2] for the epoxy resin, $10^{-15} \mathrm{~S} / \mathrm{m}$ for the LDPE [17] and $10^{-14} \mathrm{~S} / \mathrm{m}$ for the air [18]. The surface conductivity is set to be equal to the volume conductivity of the dielectric multiplied by $1 \mathrm{~km}$, which is based on a discussion of the properties of epoxy resin in [6]. In both examples the values of bulk conductivity were unimportant, the dynamics of the surface charge were dominated by surface conductivity.

In both systems symmetry boundary conditions of zero normal electric field were applied at the radial external boundaries. The electric potential was set to zero at bottom axial boundary. The electric potential at the top axial boundary is set to a $50 \mathrm{~Hz}$ AC supply with a magnitude of $10 \mathrm{kV}$.

The initial distribution of the surface charge density for the spherical void is set as the cosine of the polar angle with a peak value of $0.55 \mathrm{nC} / \mathrm{mm}^{2}$. This is based on surface charge density distributions obtained after a discharge based on simulations of the plasma dynamics [19]. The peak value was adjusted such that the electric field inside the void was significantly lower than the applied field at time zero, where the applied voltage has a magnitude of $10 \mathrm{kV}$.

For the cylindrical void the initial surface charge distribution is set as bipolar Gaussian spots. Explicitly

$$
\begin{aligned}
\sigma(u, t=0)=\sigma_{0} & \exp \left(-\frac{u^{2}}{2 s^{2}}\right) \\
& -\sigma_{0} \exp \left(-\frac{(L-u)^{2}}{2 s^{2}}\right)
\end{aligned}
$$

where $\sigma_{0}=0.13 \mathrm{nC} / \mathrm{mm}^{2}, s=0.5 \mathrm{~mm}, u$ is the distance from the point $r=0 \mathrm{~mm}, z=0.5 \mathrm{~mm}$ around the surface and $L$ is the total distance around the surface. The value of $\sigma_{0}$ was adjusted such that the electric field in the center of the void was significantly lower than the applied field at the start of the simulation.

The basis for this selection was experimental measurements performed in [20]. The applied voltage is phase shifted such that at $t=0$ the electric potential is at $-10 \mathrm{kV}$. The magnitude of the initial surface charge density distribution was adjusted such that the electric field in the void is in the same direction as the applied field, but at a much lower magnitude. This is intended to represent the condition after a discharge where the electric field has been reduced to a residual value [15]. The simulation is run for $40 \mathrm{~ms}$, which is equivalent to two applied voltage cycles.

In the implementation it was discussed that a mapping must be derived between the two dimensional model where the electrostatic equation is solved and the one dimensional model which solves for the surface charge dynamics. For the spherical void the parameterization introduced in Equation (24) is used. For the cylindrical system it was decided to parameterize the void by the arc length around the cylinder. 5 general extrusion operators were used to map each segment of the cylindrical void (3 line segments and 2 curved fillet segments), to the one dimensional model.

The distribution of the surface charge density around the surface of the spherical void is shown in Figure 6. The decay of 
charge is as expected based on the analytical calculations considered in the previous section. An unexpected result is that the surface charge density distribution undergoes a polarity inversion after the zero crossing at $5 \mathrm{~ms}$. The reason is that surface currents will always act to distribute surface charge to oppose tangential electric fields. Surface charge may be present at the boundary due to the movement of free charge carriers at the boundary, which is captured by the surface conductivity, and is unrelated to charge deployed by PDs. Of course this result is highly dependent on the value of surface conductivity which is $10^{-10} \mathrm{~S}$ in this example. The time constant associated with the movement of charge is therefore $\tau=$ $\varepsilon_{0} R / \Gamma \approx 0.044 \mathrm{~ms}$. This time constant is relatively small compared to the $20 \mathrm{~ms}$ period of the AC cycle. If a lower value of surface conductivity were used surface currents would be unable to oppose the applied field and the surface charge deployed by the PD would simply decay. While the value of surface conductivity, $10^{-10} \mathrm{~S}$, may appear unphysically high, as it results in a void which is effectively conducting and therefore unable to sustain internal PD activity, it is in agreement with values of volume conductivity quoted in the literature, [2], and the relationship between volume and surface conductivity for epoxy discussed in [6].

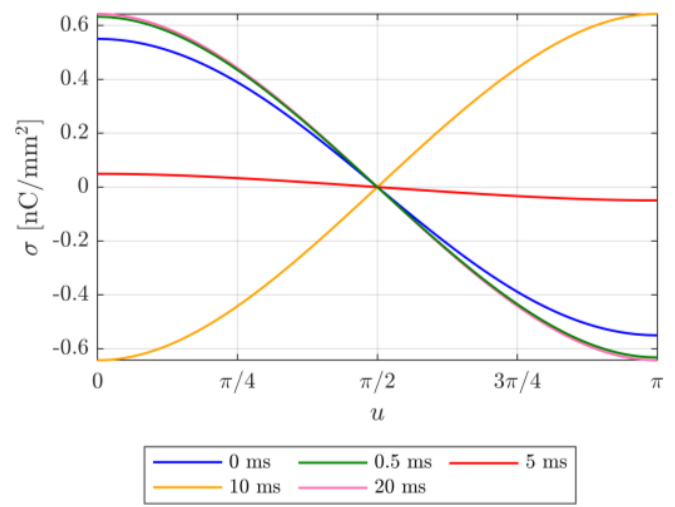

Figure 6. Surface charge density around the surface of the spherical void at different time instances.

The lower surface conductivity of the cylindrical void means the movement of surface charge has a longer time constant, approximately $10 \mathrm{~ms}$, depending on length scale selected to represent the void. This longer time constant means that the surface charge decays over the two AC cycles, as shown in Figure 7.

It is interesting that a build-up of charge is observed at the corner of the void near the fillets. This is due to a combination of two factors. Firstly, the local electric field due to initial distribution of surface charge does not generate very large tangential electric fields along the outer vertical edge of the cylinder. This causes an accumulation of charge in the vicinity of the fillets in order to generate sufficiently high local electric fields to drive surface currents along this vertical edge. The second factor is due to the applied field. The applied field will have large tangential components along this boundary, which will also lead to the accumulation of surface charge due to the movement of free charges along the boundary. It is difficult to say whether this accumulation of surface charge will inhibit or promote PD activity, as it will likely reduce the electric field while simultaneously providing a larger number of seed electrons for discharges. Surface charge accumulation near the vertical edge of cylindrical voids subject to sustained PD activity has been measured experimentally [20].

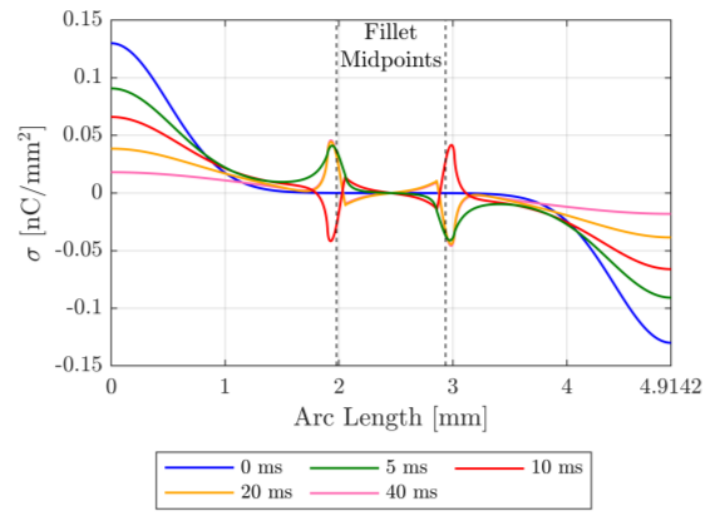

Figure 7. Surface charge density around the surface of the cylindrical void at different time instances.

High values of void surface conductivity can be caused by the formation of conductive by-products at void surfaces due to sustained discharge activity [16]. It has been demonstrated that the formation of carbonized residues can significantly impact the structure of electrical trees [21]. For surface conduction to significantly impact discharge activity over the time scale of an AC cycle requires a surface conductivity magnitude such that the time constant associated with the charge dynamics,

$$
\tau=\frac{\varepsilon_{0} l}{\Gamma}
$$

is the order of milliseconds where $l$ is a characteristic length scale of the system. High levels of surface conduction will influence phase resolved partial discharge (PRPD) patterns [15]. At sufficiently high levels of surface conductivity a void is, effectively, conducting, which will significantly inhibit PD. It should be noted that this is based on order of magnitude reasoning, for more specific insights a full model of surface charge density dynamics, solving Equation (4), is required.

\section{ELECTRIC FIELD DYNAMICS IN DC CABLE INSULATION SYSTEMS}

For AC systems the applied electric field is assumed to be determined solely by the permittivity assuming that the conductivity is insufficient to generate sufficient charge over a half-cycle. In DC insulation systems at fixed electric potential that have reached steady state the applied electric field is governed by the electrode geometry and conductivity, not the permittivity. Before reaching the DC steady state the electric field will exhibit transient behavior as it moves between a capacitive distribution, determined by the permittivity, and a resistive distribution, determined by the conductivity. In this section the applied electric field dynamics within a $1 \mathrm{~mm}$ diameter cylindrical air void in cable insulation are considered during this transient. A two dimensional slice model is used, see Figure 8.

The use of a two dimensional slice model means that electric field components along the length of the cylinder are implicitly ignored. The model is representative of the electric field distribution through the midpoint of long cylindrical voids. The electric conductivity of the XLPE insulation is assumed to be 
temperature dependent, following the work in [3]. A steady temperature gradient is present in the insulation with the inner conductor set to $60^{\circ} \mathrm{C}$ and the outer sheath set to $50^{\circ} \mathrm{C}$. To simplify the parameterization formulae the coordinate origin is set at the center of the cylinder rather the conductor. For the sake of comparison an additional model was also constructed with surface conductivity set to zero. The electric potential at the conductor is a smoothed 5 second ramp with a ramp rate of $10 \mathrm{kV} / \mathrm{s}$. The smoothing of the applied voltage profile, which is employed for numerical stability, results in a small inflection point near the start of the simulation which is visible due to the use of logarithmic axes.

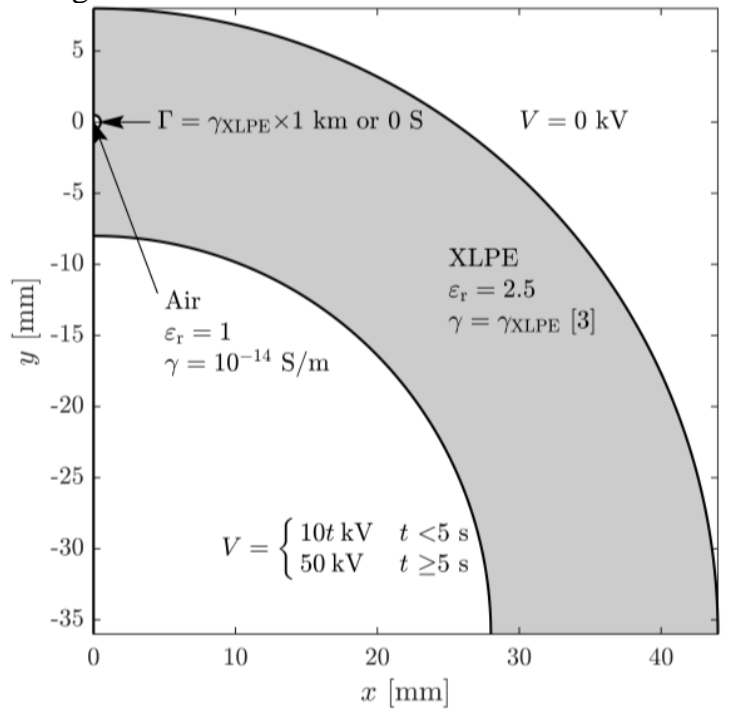

Figure 8. Model geometry of a two dimensional slice model of a cylindrical void in XLPE cable insulation. Electrical properties and the radial electric potential boundary conditions are also provided. Symmetry boundary conditions are employed on the $x=0$ axis. For clarity the lower half of the insulation annulus is excluded from the figure.

The electric field magnitude in the center of the void is shown in Figure 9 with surface conductivity neglected and considered respectively. It is immediately apparent that the surface conductivity has a significant impact on the dynamics. During the initial $5 \mathrm{~s}$ voltage ramp the surface currents rapidly oppose the change in voltage, not allowing the electric field in the void to exceed $0.015 \mathrm{kV} / \mathrm{mm}$. This is in contrast to the electric field in the void when surface conductivity is neglected, which reaches over $4 \mathrm{kV} / \mathrm{mm}$. This means that surface conductivity would prevent PD activity in the void during the ramp. When the ramp has finished surface currents act to rapidly reduce the tangential fields around the surface of the void, which effectively leads to an equipotential region with a very low electric field strength.

Over longer timescales the bulk conductivity becomes important. For the model where surface conductivity is neglected the field in void falls between $10^{3}$ and $10^{4} \mathrm{~s}$ due the higher conductivity of air compared to XLPE leading to a discontinuous normal current density $\vec{n} \cdot\left(\vec{J}_{+}-\vec{J}_{-}\right) \neq 0$. Surface charge will accumulate at the boundary, see Equation (4), even when the surface conductivity and consequently the surface current density, is zero. When the steady state is reached the normal current density each side of the boundary will be equal and, as the electrical conductivity of air is larger than that of XLPE, the electric field in the air must be lower than that in the XLPE.

When surface conductivity is considered a small rise and fall in the void electric field is observed due to the accumulation of positive space charge in the bulk insulation see Figure 9. Accumulation of space charge occurs due to the fact that the bulk XLPE conductivity is temperature dependent and therefore spatially dependent. Therefore, $\vec{\nabla} \gamma \neq \overrightarrow{0}$, which will lead to changes in volume charge density, which is initially set to zero, through Equation (19). The accumulation of space charge occurs faster in the warmer regions of the insulation, due to the higher conductivity gradient, compared to the cooler regions.

The value of surface conductivity used in these simulations is obviously critical, and in practice would need to be determined experimentally to compare against measured data. Based on the time scales of the surface charge dynamics, it is expected that for a ramp in the order of seconds the surface conductivity would have to be in the order of $10^{-14} \mathrm{~S}$ to significantly influence the electric field dynamics in the void during the ramp. For surface conduction to have a negligible impact on void field dynamics, compared to volume conduction, requires

$$
\gamma_{\text {air }} \gg \frac{\Gamma}{R}
$$

For the system under consideration this would mean that the surface conductivity would have to be significantly lower than $10^{-17} \mathrm{~S}$ for surface conduction to be insignificant compared to volume conduction.

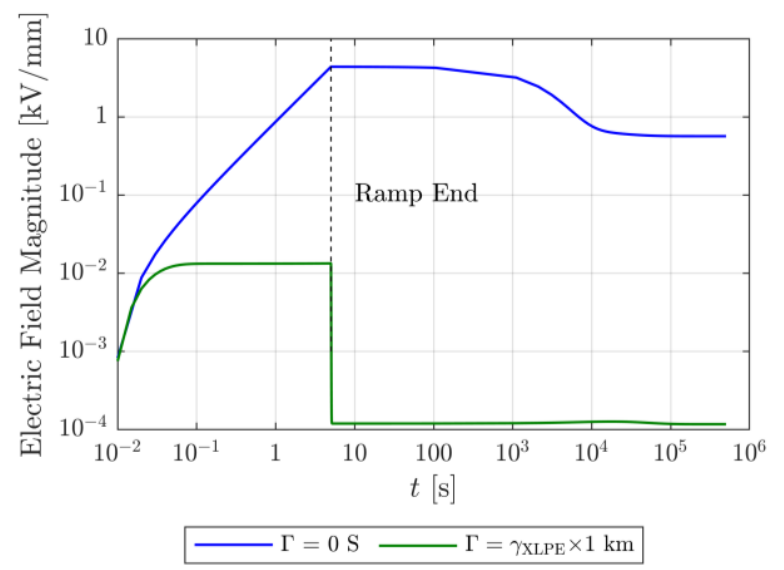

Figure 9. Electric field in the center of the cylindrical void with surface conductivity set to zero.

\section{CONCLUSIONS}

The purpose of this paper is to demonstrate how surface charge dynamics can be simulated and to illustrate the significant impact they can have on electric field distributions within insulation systems. The contributions of the paper are as follows:

- The surface divergence operator has been introduced in differential form, Equation (6), which is more amenable for numerical simulations. The calculations that are required to determine the surface metric and the contravariant tangent vectors have been introduced. This provides the framework for a numerical simulation of surface charge dynamics. 
- Earlier analytical calculations by McAllister, [5], have been used for the purposes of simulation validation. This paper serves as a reminder of this existing historical work, and stresses the need to validate model implementation.

- Surface conductivities quoted in the literature have the capability to significantly alter electric field dynamics under both $\mathrm{AC}$ and DC conditions. It should be clearly stated the theoretical background to the calculations is based on fundamental physics: electrostatics and the conservation of charge. The major uncertainty is in the input values used for electrical conductivity, both surface and volume. Careful consideration should be given to whether it is appropriate to lump together complex volume and surface charge transport into "electrical conductivities" for insulating materials.

Moving forwards it may be necessary to develop more complex models of surface charge transport using electrons and holes following space charge drift diffusion models [13]. In such cases calculating the surface divergence of a charge carrier flux would be required and could be guided by the model implementation introduced in this paper.

\section{REFERENCES}

[1] L. Niemeyer, "A Generalized Approach to Partial Discharge Modeling," IEEE Trans. Dielectr. Electr. Insul., vol. 2, no. 4, pp. 510-528, 1995.

[2] H. A. Illias, G. Chen and P. L. Lewin, "The influence of spherical cavity surface charge distribution on the sequence of partial discharge events," J. Phys. D Appl. Phys., vol. 44, no. 24, p. 245202, 2011.

[3] G. C. Montanari, P. Seri, R. Ghosh and L. Cirioni, "Noise Rejection and Partial Discharge Source Identification in Insulation System Under DC Voltage Supply," IEEE Trans. Dielectr. Electr. Insul., vol. 26, no. 6, pp. 1894-1902, 2019.

[4] I. W. McAllister, "Electric fields associated with transient surface currents," J. Appl. Phys., vol. 71, no. 7, pp. 3633-3635, 1992.

[5] I. W. McAllister, "Decay of Charge Deposited on the Wall of a Gaseous Void," IEEE Trans. Dielectr. Electr. Insul., vol. 27, no. 6, pp. 1202-1207, 1992.

[6] I. W. McAllister, "Surface Current Density K: An Introduction," IEEE Trans. Dielectr. Electr. Insul., vol. 26, no. 3, pp. 416-417, 1991.

[7] J. Kindersberger and C. Lederle, "Surface Charge Decay on Insulators in Air and Sulfurhexafluoride - Part I: Simulation,” IEEE Trans. Dielectr. Electr. Insul., vol. 15, no. 4, pp. 941-948, 2008.

[8] J. Kindersberger and C. Lederle, "Surface Charge Decay on Insulators in Air and Sulfurhexafluoride - Part II: Measurements," IEEE Trans. Dielectr. Electr. Insul., vol. 15, no. 4, pp. 949-957, 2008.

[9] S. Kumara, Y. V. Serdyuk and S. M. Gubanski, "Surface Charge Decay on Polymeric Materials under Different Neutralization Modes in Air," IEEE Trans. Dielectr. Electr. Insul., vol. 18, no. 5, pp. 1779-1788, 2011.

[10] C. Hudon, R. Bartnikas and M. Wertheimer, "Spark-to-glow Discharge Transition due to Increased Surface Conductivity on Epoxy Resin Specimens," IEEE Trans. Dielectr. Electr. Insul., vol. 28, no. 1, pp. 1-8, 1993.

[11] M. Florkowski, B. Florkowska, M. Kuniewski and P. Zydron, "Mapping of Discharge Channels in Void Creating Effective Partial Discharge Area," IEEE Trans. Dielectr. Electr. Insul., vol. 25, no. 6, pp. 2220-2228, 2018.

[12] H. F. Arnoldus, "Conservation of charge at an interface," Opt. Commun., vol. 265, no. 1, pp. 52-59, 2006.

[13] S. Le Roy, G. Teyssedre, C. Laurent, G. Montanari and F. Palmieri, "Description of charge transport in polyethylene using a fluid model with a constant mobility: fitting model and experiments," J. Phys. D Appl. Phys., vol. 39, pp. 1427-1436, 2006.

[14] E. Ildstad, "Whitehead Memorial Lecture: Challenging Defects of High Voltage Insulation Systems," IEEE Electr. Insul. M., vol. 36, no. 1, pp. 53-62, 2020.

[15] F. Gutfleisch and L. Niemeyer, "Measurement and Simulation of PD in Epoxy Voids," IEEE Trans. Dielectr. Electr. Insul., vol. 2, no. 5, pp. 729743, 1995.

[16] P. H. F. Morshuis, Partial discharge mechanisms, PhD dissertation, Dept. of Electr. Eng., Math. Comp. Sci., Delft University of Technology, NL, 1993.

[17] K. Gaska, X. Xu, S. Gubanski and R. Kádár, "Electrical, Mechanical, and Thermal Properties of LDPE Graphene Nanoplatelets Composites Produced by Means of Melt Extrusion Process," Polymers, vol. 9, no. 11, p. 11, 2017.

[18] N. Kamsalia, B. Prasadb and J. Datta, "Atmospheric electrical conductivity measurements and modeling for application to air pollution studies," Adv. Space Res., vol. 44, no. 9, pp. 1067-1078, 2009.

[19] G. Callender, I. O. Golosnoy, P. Rapisarda and P. L. Lewin, "Critical analysis of partial discharge dynamics in air filled spherical voids," J. Phys. D Appl. Phys., vol. 51, no. 12, p. 125601, 2018.

[20] K. Wu, C. Pan, Y. Meng and Y. Cheng, "Dynamic Behavior of Surface Charge Distribution during Partial Discharge Sequences," IEEE Trans. Dielectr. Electr. Insul., vol. 20, no. 2, pp. 612-619, 2013.

[21] A. S. Vaughan, I. L. Hosier, S. J. Dodd and S. J. Sutton, "On the structure and chemistry of electrical trees in polyethylene J. Phys. D Appl. Phys., vol. 39, no. 5, pp. 962-978, 2006.

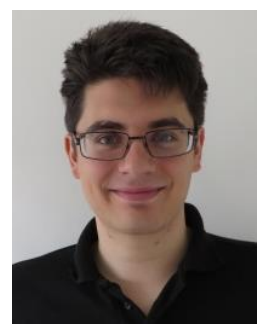

George Callender was born in Basildon, UK in 1991. He received M.Sci (Hons) in Natural Sciences (Maths and Physics) from the University of Durham, UK in 2013. He received a Ph.D. degree in electrical engineering from the University of Southampton, UK in 2018. He is currently a Research Fellow in High Voltage Numerical Modelling at the University of Southampton. His research interests include partial discharge phenomena and the thermal modelling of high voltage plant.

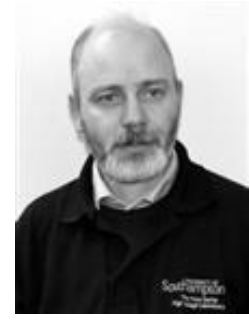

Kevin F. Goddard received a BSc in Electrical Engineering from University of Southampton, England in 1982. He obtained his PhD in 1992 for work on stray fields in the stator frames of electrical machines, also from University of Southampton.

After short periods in industry, he became a Research Fellow at University of Southampton. He worked on electromagnetic design and numerical modelling of electrical machines. His recent work involved electromagnetic and thermal modelling in cables. Dr. Goddard is a member of the IET.

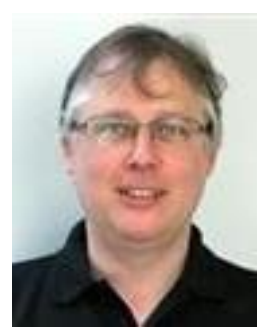

Prof. Paul L. Lewin (M'05-SM'08-F'13) was born in Ilford, Essex in 1964. He received the B.Sc. (Hons) and $\mathrm{Ph} . \mathrm{D}$. degrees in electrical engineering from the University of Southampton, UK in 1986 and 1994, respectively. He joined the academic staff of the University in 1989 and is Head of Electronics and Computer Science, where he is also Director of the Tony Davies High Voltage Laboratory. His research interests are within the generic areas of applied signal processing and control. Within high voltage engineering this includes condition monitoring of $\mathrm{HV}$ cables and plant, surface charge measurement, HV insulation/dielectric materials and applied signal processing. Since 1996 he has received funding and grants in excess of $£ 30 \mathrm{M}$, supervised 47 graduate students to successful completion of their doctoral theses and published over 500 refereed conference and journal papers in these research areas. He is a Chartered Engineer, a Fellow of the IET, and former president of the IEEE Dielectrics and Electrical Insulation Society. 